\title{
Evaluation of Local Features for Near-Uniform Scene Images
}

\author{
Tze Kian Jong \\ Faculty of Engineering \\ Universiti Malaysia Sarawak \\ Sarawak, Malaysia \\ johntkian@gmail.com
}

\begin{abstract}
Image stitching requires accurate matching of visual features to achieve good alignment. However, featurebased matching often has poor result particularly when image content is fairly near-uniform and thus it remains a challenging problem to be addressed. When the current state-of-the-art feature detectors unable to detect sufficient reliable corresponding keypoints, the output stitched images often suffer from misalignment, projective distortion and visible artefact. This paper presents a new experimental evaluation using especially near-uniform images for the performance of some well-known feature detectors, such as Harris, SIFT, SURF, BRISK and KAZE. In addition, we have also introduced $R C / S_{o}$ score to compare spatial distribution of the correct matched keypoints in overlapping region between images. The results show that the best performed local feature detector is KAZE. However, none of the tested feature detectors can reach more than $50 \%$ spread of the overlapping region.
\end{abstract}

Keywords - local features, interest points, image stitching, near-uniform scenes, quantitative evaluation

\section{INTRODUCTION}

Image stitching is basically a process of merging images with overlapping fields of view in order to obtain an image with wider field of view and higher resolution. It is fundamentally well studied in computer vision [1]. The attempt to produce image stitching with satisfactory visual results typically involve two main approaches: (1) image alignment $[2,3,4,5]$, and (2) image composition such as seam cutting [6] and blending [7]. Image alignment is considering the most crucial step in image stitching. Its execution depends very much on the task of finding precise corresponding keypoints between images of the same overlapping scene or object. When the current state-of-the-art feature detection algorithm incapable to detect sufficient and reliable correspondences, the remaining steps of image stitching will fail to deliver a pleasing stitched image and resulting in artefacts, for example misalignment or ghosting visual. It is especially obvious when image content is nearly uniform or low-texture that hardly to extract satisfactory number of correspondences in order to bring the overlapping scene into exact alignment. This is due to the homogeneous regions in near-uniform or low-texture scene images, such as sky, ocean, coast, land surface etc., that are not distinctive enough to provide rich and reliable correspondences.

In addition, irregular spatial distribution of the corresponding keypoints within overlapping region also triggering misalignment, distortion and visible artefacts in the stitching result. For instance, Fig. 1 (a) and Fig. 1 (b) depict the correct matched SIFT and KAZE features respectively between temple image pair (images obtained from [2]). Notice that both set of matched correspondences are detected in
David B. L. Bong

Faculty of Engineering

Universiti Malaysia Sarawak

Sarawak, Malaysia

bbldavid@unimas.my

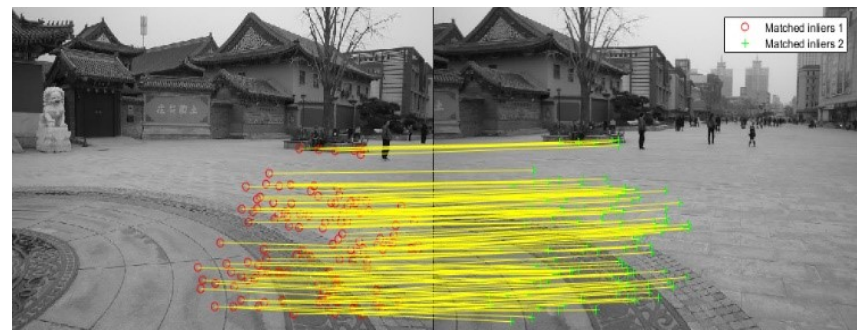

(a) Matched SIFT features

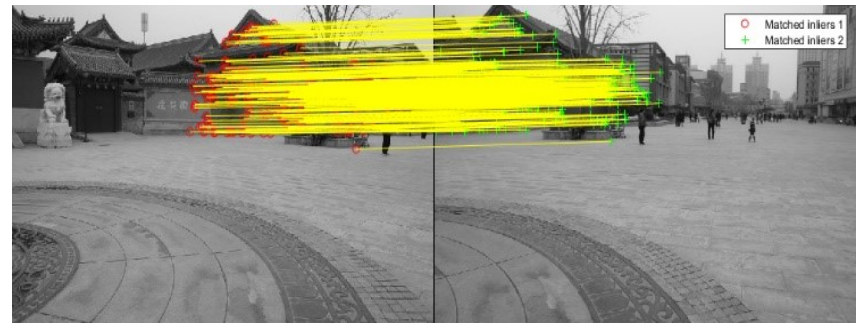

(b) Matched KAZE features

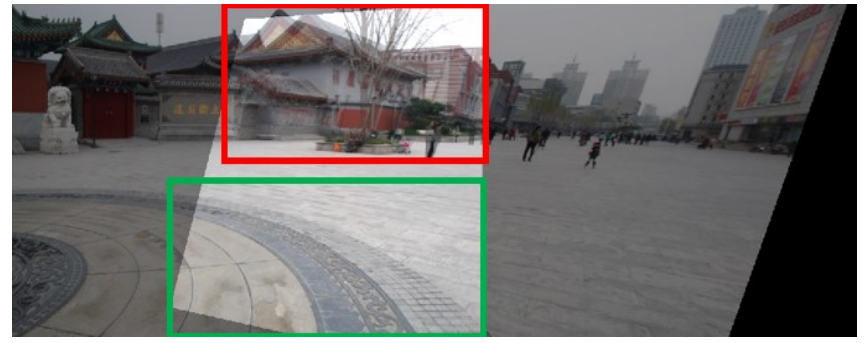

(c) Image stitching using matched SIFT features

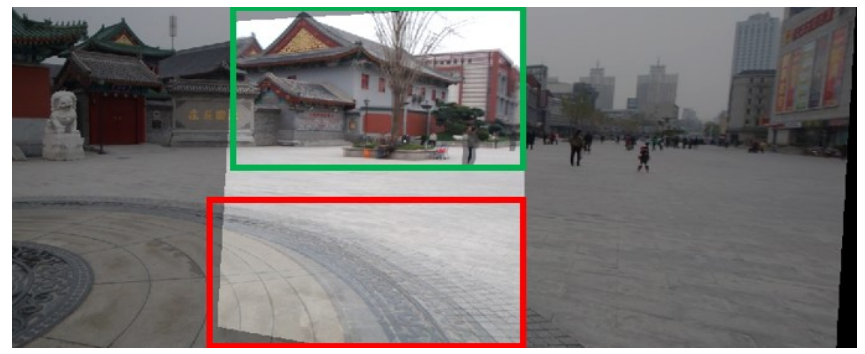

(d) Image stitching using matched KAZE features

Fig. 1. Misaligned image stitching between temple image pair due to nonuniform spread of matched correspondences within overlapping region. Red rectangles show obvious alignment errors.

different area of the overlapping region. To fit each set of data, this will lead to estimation of two unlike global homography model and consequently producing misalignment of the final stitched images. Fig. 1 (c) and Fig. 1 (d) show obvious misalignments (see red rectangles) in the stitching results. This is understandable that estimation of the global 